\title{
The Perception of Transparency in Medical Images
}

\author{
Reza Kasrai ${ }^{1,2}$, Frederick A.A. Kingdom ${ }^{2}$, and Terry M. Peters ${ }^{1,3}$ \\ 1 McConnell Brain Imaging Ctr., Montreal Neurological Inst., Canada H3A 2B4 \\ 2 McGill Vision Research Unit, Royal Victoria Hospital, Montreal, Canada, H3A 1A1 \\ ${ }^{3}$ Imaging Research Labs., Robarts Research Institute, London, Canada, N6A 5K8 \\ rkasrai@bic.mni.mcgill.ca \\ fred@jiffy.vision.mcgill.ca \\ tpeters@irus.rri.on.ca
}

\begin{abstract}
Many of the tasks performed by clinicians during surgical planning or diagnosis rely on the visualization of medical images. When information from multiple modalities is integrated, some images must be seen through others. In these cases, surfaces or volumetric images are rendered semi-opaque, or transparent. It is necessary, therefore, to understand the nature of transparency perception in human visual system. A set of experiments is presented which begins to look at the role of stereoscopy, spatial frequency, and multiple layers in the perception of transparent achromatic surfaces. An adjustment technique has been developed for the measurement of observer performance, and a physical and algebraic model for the perception of multiple transparent surfaces is described. The results show good agreement with the models developed.
\end{abstract}

\section{Introduction}

With the continual increase in the speed of relatively affordable computers, and the rise in the number of their applications to medicine, the question of how well computers and algorithms perform is being replaced by one examining the nature of the interaction of humans with machines. Although computers are now involved in various aspects of medicine, in many cases the output of algorithms is in the form of images. These images are subsequently used by clinicians for the purpose of diagnosis or surgical planning. It stands to reason then, that when users make decisions about surgical procedures or courses of treatment based on images, that these images convey not only the correct information, but that they represent it in a manner that an observer can best understand.

Recent visualization systems allow the registration and integration of images from many different imaging modalities, in order to provide the maximum amount of information to the clinician. 2-dimensional (2D) sections of volumetric anatomical data (e.g. MRI, CT) are typically overlaid with the corresponding functional or chemical (EEG, PET, fMRI, MRS) images, such that the structural image can be 'seen through' the functional image. From the same volumetric data, surfaces corresponding to structures of interest are segmented out. 
In many cases, a surface is enclosed within, or is transected by, another surface, such as when viewing a thalamotomy lesion target (typically the vim-one of the thalamic nuclei) through the wall of the thalamus [1], or when visualizing virtual radiofrequency lesions enveloping a target structure (Figure 1$)^{1}$. In all these situations, the surface or reconstructed slices simulated to be closer to the observer are rendered as semi-opaque, or transparent ${ }^{2}$. An early example of multi-modality integration of 3D data, is shown in Figure 3, where a volume rendering of the distal hemisphere is overlaid by a co-registered digital subtraction angiogram (DSA) of the proximal hemisphere.

While the number of such rendering algorithms is ever increasing, little or no work is dedicated to the rigorous psychophysical study of the nature of the interaction of the human visual system with the images generated. Clearly, given the complexity and richness of medical images, especially those integrating multiple imaging modalities, this is not a simple task. Nevertheless, an extensive literature exists in the realm of psychophysics, exploring different aspects of human visual perception, which provides the beginnings of a course of investigation.

The aim of this paper is to begin to bridge the gap between the fields of computer graphics, medical image visualization, and human vision research, using the techniques of experimental psychology, by introducing a series of experiments focusing on specific aspects of the perception of transparency. The purpose of these studies is twofold: to understand how and to what degree of accuracy properties of transparent surfaces (reflectance, opacity, color, texture, etc.) and opaque surfaces seen through transparent ones are encoded, and subsequently to apply these results to medical images, in order to optimize and facilitate the transmission of relevant information to clinical users, especially in the context of image-guided neurosurgery.

\section{Related Work}

\subsection{Vision Literature}

Work on the perception of transparency began in earnest following the first in a series of papers by Metelli [3,4]. He developed an algebraic formulation based on Talbot's law of color fusion, $z=\alpha x+(1-\alpha) y$, where $0 \leq \alpha \leq 1$ is the linear mixing proportion of the achromatic colors $x$ and $y$, as measured by their reflectances, resulting in the color $z$. This formulation is similar to the "blending" function used in computer graphics [6], whence the term alphablending. By analogy, the color of a pixel $p$, which is the combination of a background surface $\mathbf{A}$, seen through a transparent surface $\mathbf{P}$, is given by $p=t a+$ $(1-t) r$, where $a$ is the reflectance of surface $\mathbf{A}$, and $t, r \in[0,1]$ the transmittance

\footnotetext{
${ }^{1}$ In this case, the surface was segmented out of a volumetrically defined digital atlas of the human brain.

2 The term transparent, as opposed to translucent, is used to imply that the image is not distorted by the overlying surface. Transparency is also the term conventionally used in vision literature.
} 
and reflectance of surface $\mathbf{P}$. The assumption here is that the absolute values of $r$ and $t$ can be somehow calculated or encoded by the visual system given the four reflectance values, $a, b, p$, and $q$, and also that both the background and transparent surfaces are illuminated identically.

Although matching tasks with stimuli generated using this model have produced very good results in human observers [4], limiting the values of $t$ and $r$ to between 0 and 1 has been shown to be too restrictive [7], leading to a modified set of equations based on luminances rather than reflectances $[8,9]$. This modification allows for the possibility of inhomogeneous illumination:

$$
P=t a I+(1-t) r I^{\prime}=t A+(1-t) r h I=t A+F,
$$

where $P$ and $A$ are now luminances (e.g. in $c d / m^{2}$ ), $I$ and $I^{\prime}$ the (non-equal) illumination components for the two surfaces with ratio $h$, such that $I^{\prime}=h I$. The second term is collapsed into an overall additive component $F$, since with four known luminances $(A, B, P, Q)$ and two equations, only two unknowns, $t$ and $F$, can be extracted, and the product $r h I$ cannot be disambiguated without additional assumptions or a priori knowledge.

\section{$2.2 \quad$ Imaging Literature}

In a review of the cues relevant to the visualization of medical images, Kundel [10] wrote, "No matter what the task or the image, as long as an observer is needed to read out the information, performance is the final arbiter of the goodness of the image." This implies that regardless of what may be considered a set of important features in a medical image for a given task (high contrast, texture, lighting, etc.), human performance, measured if possible against some known gold standard or truth, must be the determining measure, since it is after all the human system for which the images are generated.

Even though the latter may be considered a truism, very few developers of visualization software actually set out to evaluate rigorously the sensitivity and accuracy of a given rendering technique. More typically, validation is described in terms of clinical utility as reported anecdotally by a clinician, or in the context of a limited number of cases where a diagnosis is confirmed by biopsy or at surgery $[11,12]$. Part of the problem may lie in the complexity or non-specificity of the task, in that no algorithm or software can be made optimal for all tasks, or range of tasks. In addition, it is often difficult to find a large number of sufficiently similar clinical cases where the 'true' diagnosis is known. As a result, compromises are made, or the software is designed with enough flexibility that users can choose a rendering method or a segmentation algorithm which they believe suits the task [13]. It is nonetheless possible to conduct meticulous studies to show the superiority of one human-machine system over another using well-developed statistical methods, such as receiver operating characteristic curves [14] or simple analysis of variances. 


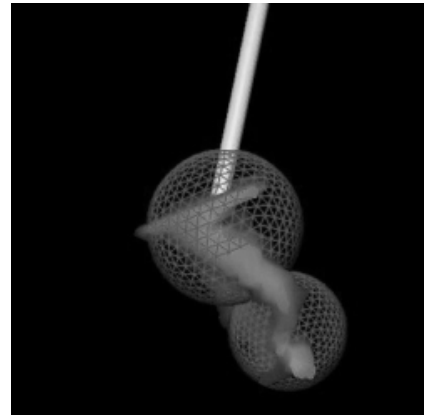

Fig. 1. Simulated spherical rf lesions encompassing the vim nucleus of the human thalamus.

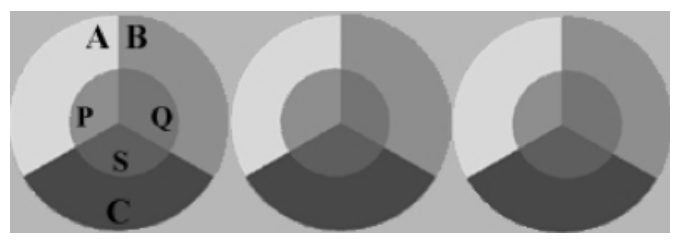

Fig. 2. Stereo transparecy stimuli arranged for crossed-eye viewing. Crossing the right pair produces a positive disparity (in front) percept, and the left pair gives the negative (behind) percept.
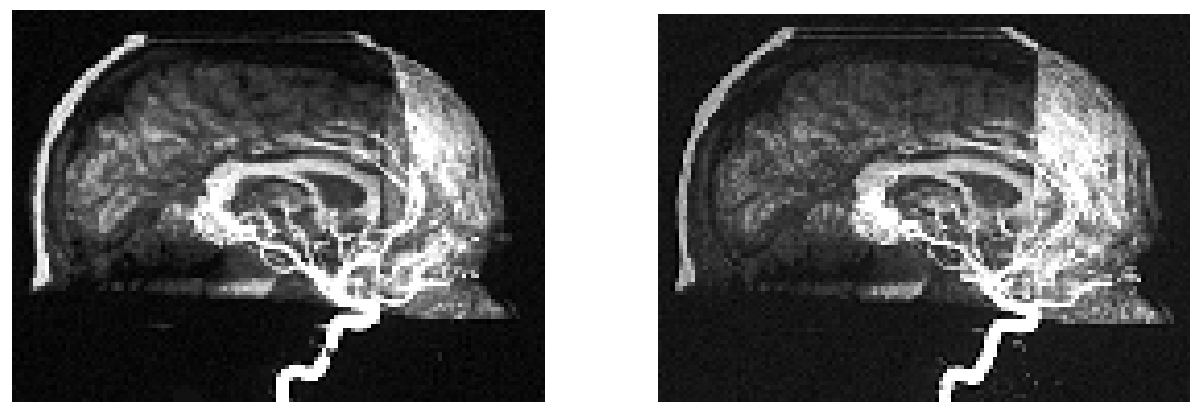

Fig. 3. Volume-rendered MRI with overlaid DSA, arranged for crossed-eye viewing [2].
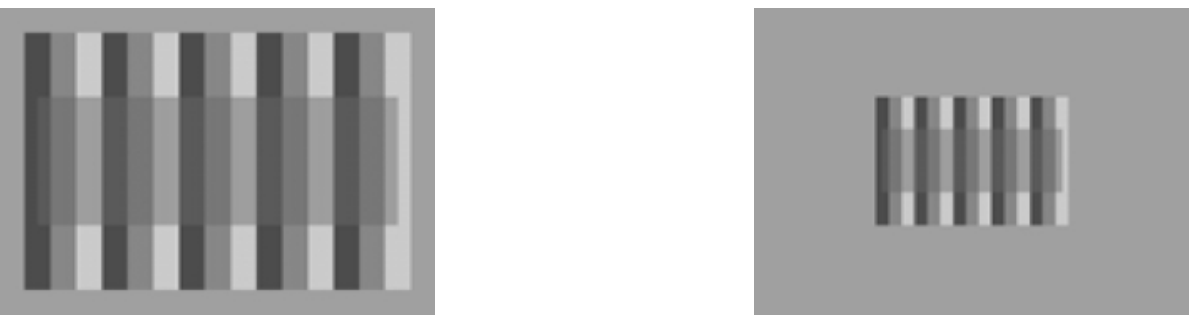

Fig. 4. Spatial frequency stimuli. 


\section{Methods}

\subsection{General}

All experiments were performed using an SGI (Silicon Graphics Inc., Mountain View, CA, USA) O2 workstation (150 MHz R10000 Processor) on a 17" monitor displaying 1280x1024 pixels at a vertical refresh rate of $72 \mathrm{~Hz}$. The luminance output of the monitor was measured using a single channel optometer with photometric detector (Model S370, United Detector Technology), and calibrated such that desired luminances between 0 and $\approx 40 \mathrm{~cd} / \mathrm{m}^{2}$ could be reliably reproduced. Stereoscopic image pairs (maximum size of $9 \times 9 \mathrm{~cm}^{2}$ each) were viewed through a custom-built 8-mirror stereoscope with a principle ray path of $45 \mathrm{~cm}$, for a maximum visual angle of $11.4^{\circ}$. Subjects adjusted the luminance of test patches using the arrow keys on a standard keyboard, and pressed a separate key to end the trial once they were satisfied with their adjustment. Responses were recorded to a text file for later analysis.

\subsection{Generation of Stimuli}

Transparency in Stereo A six-luminance stimulus, originally described by Gerbino (unpublished results; personal communication, 1999), was generated (Figure 2). The stimulus consisted of two concentric circles each divided into three equal overlapping sectors, producing an illusory transparent layer on a tri-partite background, such that the sectors with luminances $P, Q$, and $S$ were 'on top' of the luminances $A, B$, and $C$. The layer luminances $P$ and $Q$ were calculated according to the luminance transparency model (Equation 1). The subjects' task in all conditions was to adjust the luminance $S$ (set to a random value at the beginning of each trial) such that the transparent layer formed a contiguous disk with uniform transmissive and reflective characteristics on the tri-partite background. For each trial a stereo-pair was presented so that the inner circle simulating a transparent layer was disparate with respect to the outer circle. Three different conditions were produced under which the disparity was less than, equal to, or greater than zero, such that the layer was stereoscopically defined as being respectively behind, coplanar with, and in front of the background circle. Subjects' adjustments of the luminance $S$ were compared to the theoretically expected luminance, $S=t C+F$, as calculated according to the model. The luminance of the area surrounding the stimulus was fixed at an intensity of half the maximum luminance produced by the display (i.e, $20 \mathrm{~cd} / \mathrm{m}^{2}$ ). Values of $A, B, C$, and $F$ were generated randomly, along with five different values of $t(0.1,0.3,0.5,0.7,0.9)$ with the constraints that $\{A, B, C\} \neq 20 \mathrm{~cd} / \mathrm{m}^{2}$ (surround color), and $\{A, B, C, P, Q, S\}<40 \mathrm{~cd} / \mathrm{m}^{2}$ (the maximum luminance output). A set of 40 different stimuli were produced and presented for the three disparity conditions.

Transparency and Spatial Frequency A stimulus consisting of a variegated background overlaid with an illusory horizontal transparent strip was generated 
using the same combination of six luminances (Figure 4). The spatial frequency content of the stimulus was manipulated either by changing the size of the stimulus or by changing the viewing distance.

Multiple Surface Transparency Following by analogy from the single layer transparency model of Equation 1, a model for two layer transparency was devised, allowing for different illuminations of each layer. The optic array corresponding to the model is shown in Figure 5. If layer $1\left(t_{1}, r_{1}\right)$ is above layer 2 $\left(t_{2}, r_{2}\right)$, the contributions from each layer sum to give the theoretical luminance,

$$
Z=t_{2} t_{1} a I+t_{2}\left(1-t_{1}\right) r_{1} I^{\prime}+\left(1-t_{2}\right) r_{2} I^{\prime \prime}
$$

or more simply,

$$
Z=t_{2} t_{1} A+t_{2} F^{\prime}+F^{\prime \prime}
$$

where $F^{\prime}$ and $F^{\prime \prime}$ are defined as before for simplification. It can be easily shown that if layer 1 is on top of layer 2, the indices are simply reversed, such that,

$$
Z=t_{1} t_{2} A+t_{1} F^{\prime \prime}+F^{\prime}
$$

This implies that, in the absence of any binocular cues, for given values of $t_{1}, t_{2}, F^{\prime}$, and $F^{\prime \prime}, Z$ can take two different values, depending on the depth order of the layers. $Z$ is uniquely defined, however, when disparity information is available in stereo pairs. A square $(\mathbf{A})$ was overlaid with two orthogonal transparent strips, simulated to have different transmissive and reflective properties, such that the region of overlap $(\mathbf{Z})$ was a square patch in the center of the square (Figure 6). The two strips were presented in stereo depth relative to the background square, such that one of the strips $\left(t_{1}, r_{1}\right)$ was between the background square and the other strip $\left(t_{2}, r_{2}\right)$. The depth order of the strips was randomized. The subjects were to adjust the luminance $Z$ of the central patch such that it was consistent with the properties of both strips, including their relative depth.

\section{Results}

Figure 7 shows the lines of best fit to the data under the three disparity conditions for one subject (RK). The correlation coefficients $\left(r^{2}\right)$ for the no disparity, the positive disparity, and negative disparity conditions were 0.94, 0.95, and 0.85 , respectively. In addition, the root-mean-square (RMS) residuals for each condition were calculated. For the no disparity, the positive disparity, and negative disparity conditions, respectively, the RMS residuals were $1.49,1.45$, and $2.37 \mathrm{~cd} / \mathrm{m}^{2}$ with standard deviations of $1.51,1.42$, and $2.38 \mathrm{~cd} / \mathrm{m}^{2}$. RMS residuals as a function of spatial frequency for two subjects (RK, FK) are shown in Figure 8. Each point on the graph represents the RMS residual for a set of 40 trials. Figure 9 shows the regression parameters for the set of 40 doubletransparency adjustments by one subject (RK). 


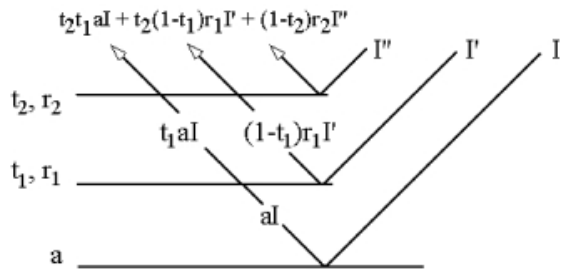

Fig. 5. Optic array for two transparent layers.

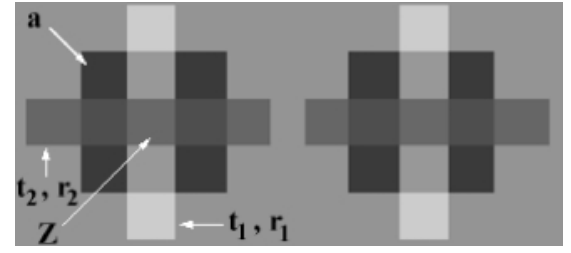

Fig. 6. Double transparency stimulus (stereo-pair).

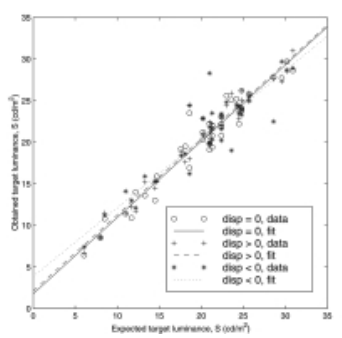

Fig. 7. Results for stereo transparency conditions.
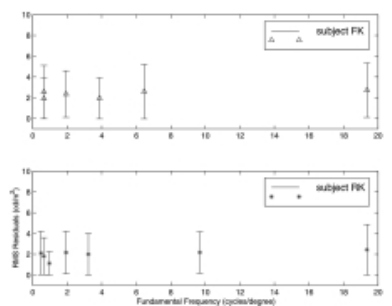

Fig. 8. Root-meansquare residuals (with standard deviation error bars) for spatial frequency experiments.

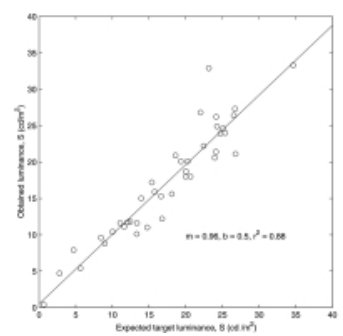

Fig. 9. Regression through doubletransparency data.

\section{Conclusions}

Even though the results presented here are preliminary, we may derive a number of significant ideas. The stereo transparency correlation coefficients show that the model accounts for better than $94 \%$ of the variance in the data under ecologically valid conditions (flush and in-front disparities). A transparent surface, on the other hand, would not be expected to be visible through an opaque one-a condition which is simulated when negative disparity stimuli are presented. As a result, the RMS residual is higher than the other two conditions and the correlation coefficient lower. This effect is corroborated by observers of early multiple modality renderings, where rivalrous depth cues (occlusion/transparency and binocular disparity) produce competing or uncomfortable percepts. Above all, the zero- and positive-disparity results are a validation of the luminance transparency model. The measurement technique described is quite simple, considering only six distinct luminances are used. As such it may be modified such that stimuli which incorporate more of the complexity of medical images are employed in similar studies. The results showing subjects' performance as a function of spatial frequency content show no definitive trends. Although one might conclude from the figures that spatial frequency plays no role in transparency perception, the effects at higher frequencies remain to be seen. Clearly 
as the resolution limit of the eye is approached, one would expect a degradation in performance. Unfortunately the resolution of the display was a limiting factor at the viewing distances utilized in these experiments. Studies exploring these higher frequencies are already underway, since it would be interesting to measure whether 'transparency acuity' follows a trend similar to the contrast sensitivity function. In addition, if a maximum sensitivity in transparency adjustments within a particular frequency band can be found, it might correspond to an ideal surface texel (texture element) size. The double layer results show good agreement with the model, indicating the optic array of Figure 5 is a reasonable one, not only for two transparent layers, but perhaps extending to multiple layers.

\section{References}

1. P. St-Jean, A. F. Sadikot, L. Collins, D. Clonda, R. Kasrai, A. C. Evans, and T. M. Peters, Automated atlas integration and interactive three-dimensional visualization tools for planning and guidance in functional neurosurgery, IEEE Trans Med Imaging, vol. 17, pp. 672-80, 1998. 727

2. D. L. Collins, Volumetric Rendering of Medical Data, Ph.D. Thesis, Montreal, Canada: McGill University, 1990. 729

3. F. Metelli, The perception of transparency, Sci Am, vol. 230, pp. 90-8, 1974. 727

4. F. Metelli, O. Da Pos, and A. Cavedon, Balanced and unbalanced, complete and partial transparency, Percept Psychophys, vol. 38, pp. 354-66, 1985. 727, 728

5. M. D'Zmura, P. Colantoni, K. Knoblauch, and B. Laget, Color transparency, Perception, vol. 26, pp. 471-92, 1997.

6. M. Woo, J. Neider, and T. Davis, in OpenGL programming guide: the official guide to learning OpenGL, version 1.1: Addison-Wesley Developers Press, 1997, pp. 21326. 727

7. J. Beck, K. Prazdny, and R. Ivry, The perception of transparency with achromatic colors, Percept Psychophys, vol. 35, pp. 407-22, 1984. 728

8. W. Gerbino, C. I. Stultiens, J. M. Troost, and C. M. de Weert, Transparent layer constancy, J Exp Psychol Hum Percept Perform, vol. 16, pp. 3-20, 1990. 728

9. W. Gerbino, Achromatic Transparency, in Lightness, Brightness, and Transparency, A. L. Gilchrist, Ed.: Lawrence Erlbaum Associates, 1994, pp. 215-255. 728

10. H. L. Kundel, Visual cues in the interpretation of medical images, J Clin Neurophysiol, vol. 7, pp. 472-83, 1990. 728

11. R. A. Robb, S. Aharon, and B. M. Cameron, Patient-specific anatomic models from three dimensional medical image data for clinical applications in surgery and endoscopy, J Digit Imaging, vol. 10, pp. 31-5, 1997. 728

12. P. Kay, R. Robb, R. Myers, and B. King, Creation and validation of patient specific anatomical models for prostate surgery planning using virtual reality, presented at Visualization in Biomedical Computing, 4th International Conference, Berlin, Germany, 1996. 728 
13. G. D. Rubin, M. D. Dake, S. Napel, R. B. Jeffrey, Jr., C. H. McDonnell, F. G. Sommer, L. Wexler, and D. M. Williams, Spiral CT of renal artery stenosis: comparison of three-dimensional rendering techniques, Radiology, vol. 190, pp. 181-9, 1994. 728

14. C. E. Metz, ROC methodology in radiologic imaging, Invest Radiol, vol. 21, pp. 720-33, 1986. 728 